\title{
Video Motion for Every Visible Point
}

\author{
Susanna Ricco and Carlo Tomasi \\ Duke University \\ Durham, NC, USA \\ $\{$ sricco, tomasi\}@cs.duke.edu
}

\begin{abstract}
Dense motion of image points over many video frames can provide important information about the world. However, occlusions and drift make it impossible to compute long motion paths by merely concatenating optical flow vectors between consecutive frames. Instead, we solve for entire paths directly, and flag the frames in which each is visible. As in previous work, we anchor each path to a unique pixel which guarantees an even spatial distribution of paths. Unlike earlier methods, we allow paths to be anchored in any frame. By explicitly requiring that at least one visible path passes within a small neighborhood of every pixel, we guarantee complete coverage of all visible points in all frames. We achieve state-of-the-art results on real sequences including both rigid and non-rigid motions with significant occlusions.
\end{abstract}

\section{Introduction}

The goal of long-range, high-density motion estimation in video analysis is to compute the life of every point in a dense sampling of the visible surfaces in the scene. The image projection of a scene point moves along a path in the image plane. Sometimes the point is visible, and sometimes it is occluded by some object in the world or by the boundaries of the image. In a dense motion estimate, at least one path passes through every pixel of the sequence.

Dense, long-range motion estimation supports a number of applications. The computed paths can propagate to multiple frames any annotations or edits made in a single frame, thereby easing video labeling and editing. If visible paths can be extrapolated into regions where they are occluded, the occluding object can be removed from the video by painting the pixels it occupies with the extrapolated colors. Videos can be segmented into separate objects by clustering paths into coherent groups. The shapes and appearance of the resulting tube-like regions can support the detection and recognition of objects and activities.

Image motion information is either poor or altogether
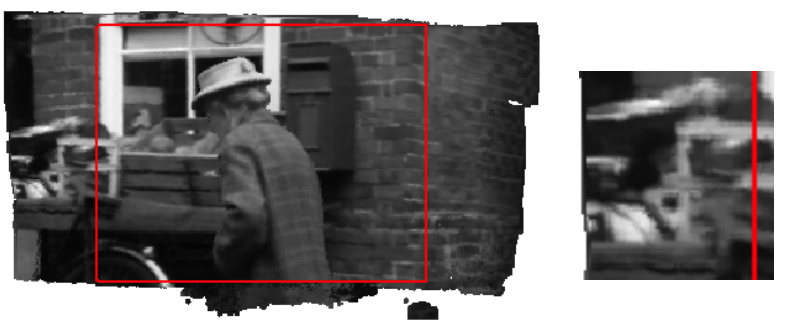

(a) Our approach.
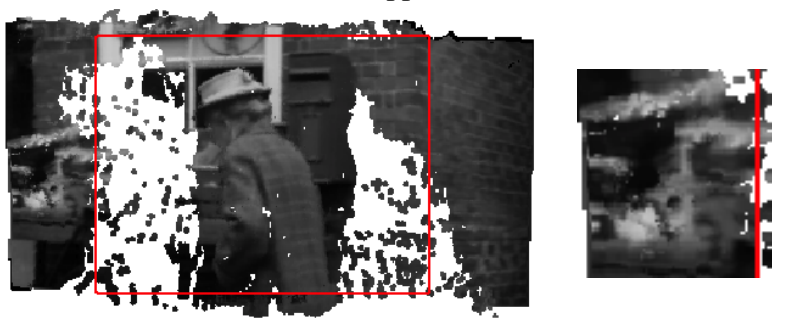

(b) Lagrangian motion [15].

Figure 1: Top: Result of transporting all gray levels in the 25 -frame marple 7 sequence to frame 13 by the image motion computed with our method. The camera pans to follow Miss Marple as she walks from right to left. Pixels inside the red rectangle are native to frame 13 . We find motion in all regions visible in any frame. Lagrangian motion (b) only computes paths for points visible in the first or last frame. The missing crate under the window is behind Miss Marple in the last frame and off-screen in the first. The missing portion of the wall to the right of the mailbox is behind Miss Marple in the first frame and off-screen in the last. Details on the right highlight regions where incorrect estimates in Lagrangian motion create artifacts which we avoid.

unavailable where the scene has little or no visual texturethe so-called aperture problem. As a consequence, regularization - or priors in probabilistic parlance-must be employed to extrapolate motion information from textured to poorly textured regions. To this end, we assume that (i) image paths live in a low-dimensional space, (ii) appearance 
remains approximately constant along the visible portion of a path, and (iii) exactly one world point is visible at every image point. The first assumption is exactly satisfied with rigid motion, and approximately satisfied in many circumstances. The second assumption is pervasive in motion analysis, and the third excludes semi-transparent objects.

\section{Summary of Contributions}

Our formulation is related to the concept of Lagrangian Motion Estimation (LME) proposed by Ricco and Tomasi [15]. Like them-and several others-we assume that paths belong to a low-dimensional subspace. We also anchor each path to a single pixel in the sequence, so as keep paths from bunching up. Similarly to LME, we also describe path visibility with a binary, per-frame flag, and cast motion estimation as energy minimization.

However, our method differs in important ways from LME. First, we do not have fixed "reference frames" to anchor paths into. In LME, this limitation restricts estimated paths to only those that are visible in either the first or last frame of the video. Paths born after the first frame or that die before the last are never found in LME, while we find paths wherever they are visible. Figure 1 illustrates this.

Second, we minimize the energy function by direct nonlinear optimization rather than by solving Euler-Lagrange PDEs. The greater flexibility of our method allows for both anchors in any frame-a generalization that seems inherently infeasible in the LME approach - and more realistic regularization functionals leading to more accurate paths. The details in the right column of Figure 1 show an example where the reconstructed image found with our method does not exhibit the artifacts visible in the LME results.

Third, we formulate the computation of the visibility flag as a Maximum a Posteriori (MAP) Markov Random Field (MRF) estimation problem, for which an efficient solution method is available. This formulation allows for the explicit enforcement of the constraint that there must be exactly one visible path at every pixel. In contrast, LME's real-valued relaxation method for this combinatorial optimization problem approximates the target function, and leaves pixels unexplained.

\section{Related work}

Decades of research into motion estimation has focused primarily on the computation of optical flow fields between consecutive frames. Here, we consider approaches that compute longer paths.

Sundaram et al. [18] concatenate flow fields found by Large Displacement Optical Flow (LDOF) [4] into longer paths, each computed independently of the others. Their paths start in regions with sufficient texture, but cover more image regions than feature trackers like KLT [13] do. Paths end at detected occlusion and motion boundaries, found by comparing flow fields computed forward and backward in time. Sand and Teller [17] start with concatenated optical flow vectors but refine these post facto by optimizing a cost function with multiframe data and smoothness terms. Highcost paths end at suspected occlusions, and new ones are started to fill gaps.

Early formulations for temporal regularization penalize changes in image velocity in both time and space [21]. Structure-from-motion methods regularize more globally by assuming rigid motion - a restrictive assumption-for which image paths can be proven [19] to lie in a space of low and known dimension. This work has been extended to multiple rigid motions [8] and to non-rigid motion [3, 1$]$. These techniques precompute paths with frame-to-frame trackers, and de-noise them post facto by projection into a low-dimensional subspace.

More recent methods apply subspace constraints during path estimation to track points that are difficult for a frame-to-frame tracker to follow. Early approaches applied subspace constraints during optical flow estimation to improve estimates in untextured regions of rigid scenes [11] or sampled from a path subspace to improve motion estimates along intensity edges affected by the aperture problem [20]. Garg et al. [9] combined subspace constraints with variational techniques adapted from optical flow estimation to solve for the multiframe registration of deforming surfaces. They compute full-length paths for every point in a selected reference frame. An extension softens the subspace constraint to create a prior on image motion [10]. These methods do not handle occlusions, limiting their applicability.

LME finds paths by optimizing a global energy function over the entire video. It models visibility explicitly, and reconnects paths across brief occlusions. As explained earlier, we improve upon LME by removing its reliance on reference frames, handling visibility combinatorially rather than by approximate relaxation, and minimizing energy by direct optimization rather than variational methods. Our extension has the benefits mentioned in the introduction and demonstrated by the results in Section 6.

\section{Model}

Let $p$ be an index into a set of paths $\boldsymbol{x}_{p}(t): \mathcal{T} \rightarrow \mathbb{R}^{2}$, where $\mathcal{T}$ is the (discrete) time domain of the video sequence. A path is visible at time $t$ iff its visibility flag $\nu_{p}(t): \mathcal{T} \rightarrow\{0,1\}$ is equal to 1 at time $t$. Both functions $\boldsymbol{x}_{p}(t)$ and $\nu_{p}(t)$ are unknowns to be estimated for all paths in a given video sequence. To ensure approximately (at first, and exactly later) at least one path per pixel in every frame, we anchor $\boldsymbol{x}_{p}(t)$ to point $\boldsymbol{u}_{p}$ in some frame $\tau_{p}$ by letting $\boldsymbol{x}_{p}\left(\tau_{p}\right)=\boldsymbol{u}_{p}$, and require enough anchor points to have some path pass through every pixel in the video sequence. In contrast with LME, $\tau_{p}$ is path-specific and unrestricted.

Paths are assumed to be in the space spanned by a 
sequence-specific basis of paths $\left\{\varphi_{1}, \ldots, \varphi_{K}\right\}$, up to a shift:

$$
\boldsymbol{x}_{p}(t)=\boldsymbol{u}_{p}+\sum_{k=1}^{K} c_{p k}\left(\boldsymbol{\varphi}_{k}(t)-\boldsymbol{\varphi}_{k}\left(\tau_{p}\right)\right) .
$$

The motion relative to the anchor point $\boldsymbol{x}_{p}\left(\tau_{p}\right)=\boldsymbol{u}_{p}$ is determined by the unknown coefficients $\boldsymbol{c}_{p}=\left(c_{p 1}, \ldots, c_{p K}\right)$.

Since paths in a video with $F$ frames have $F$ points, the standard basis over $\mathbf{R}^{2 F}$ can represent any path exactly. However, for many sequences a much more compact $(K<<2 F)$ basis is adequate, and provides powerful, sequence-specific regularization.

Given basis paths and anchor points, we find paths and visibility flags by interleaving computing optimal paths given visibility with computing optimal visibility given paths. The next two sections define the optimality criteria for these computations. Section 4 shows how to find the path basis and initial anchors, and Section 5 shows how to compute optimal paths, visibility, and anchors.

\subsection{Optimal paths}

Given a set of basis paths and a set of anchors, we find the best motion coefficients for each path by minimizing an objective function that penalizes changes in appearance along a path (temporal smoothness) and differences between nearby paths (spatial smoothness):

$$
\sum_{p \in \mathcal{P}} \sum_{t=1}^{F} E_{D}\left(\boldsymbol{c}_{p}, t\right)+\lambda \sum_{p, q \in \mathcal{P}} E_{S}\left(\boldsymbol{c}_{p}, \boldsymbol{c}_{q}\right) .
$$

The first term,

$$
E_{D}\left(\boldsymbol{c}_{p}, t\right)=\nu_{p}(t) \Psi\left(I\left(\boldsymbol{c}_{p}, t\right)-I\left(\boldsymbol{c}_{p}, \tau_{p}\right)\right),
$$

employs a robust penalty function $\Psi(s)=\sqrt{s^{2}+\epsilon^{2}}$ to measure the difference between the image intensity $I\left(\boldsymbol{c}_{p}, t\right)=I\left(\boldsymbol{x}_{p}(t)\right)$ of the path in frame $t$ and that at the anchor $\boldsymbol{u}_{p}$ in frame $\tau_{p}$. Multiplication by $\nu_{p}(t)$ ensures that this penalty is levied only on visible points. The second term,

$$
E_{S}\left(\boldsymbol{c}_{p}, \boldsymbol{c}_{q}\right)=\alpha_{p q} \sum_{k=1}^{K} \Psi\left(c_{p k}-c_{q k}\right),
$$

measures the difference between the motion coefficients of pairs of paths. The multiplier $\alpha_{p q}$ couples nearby paths that have similar appearance, and is equal to

$$
\alpha_{p q}=\exp \left(-\frac{\left(I\left(\boldsymbol{c}_{p}, \tau_{p}\right)-I\left(\boldsymbol{c}_{q}, \tau_{q}\right)\right)^{2}}{\sigma^{2}}\right)
$$

if the path $p$ is visible in the anchor frame of path $q$ (that is, if $\left.\nu_{p}\left(\tau_{q}\right)=1\right)$ and passes close enough to the anchor of $q$ (that is, if $\left\|\boldsymbol{x}_{p}\left(\tau_{q}\right)-\boldsymbol{u}_{q}\right\|<\Delta$ ). Otherwise, $\alpha_{p q}=0$.

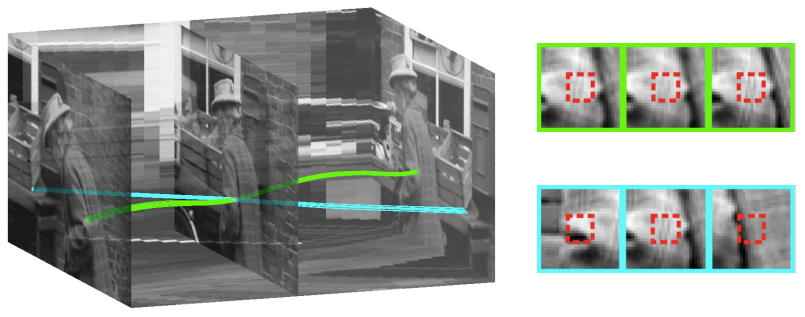

Figure 2: A spatiotemporal cube of the marple7 sequence. Time runs from left to right. The corner of the crate (cyan) is first occluded by Miss Marple's arm (green) in frame 12. A small patch (red dashed squares) around each path in every frame is transported along the current path estimates and monitored for consistent appearance. The arm patch (top right) is most consistent, and makes this the controlling path at that point and frame. Points along paths that either coincide with or are substantially parallel to a nearby controlling path have their observed visibility flag $\hat{\nu}_{p}(t)$ set to 1 . All other flags are set to 0 . Observed flags affect the estimated visibility flags at the nodes of a MRF that enforces spatial and temporal consistency of the flags and ensures that at least one path is visible at every pixel.

\subsection{Optimal visibility}

The binary visibility flag $\nu_{p}(t)$ for each path and frame is modeled as a MRF whose structure depends on the current estimates $\boldsymbol{x}_{p}(t)$ of the paths $p \in \mathcal{P}$. The MRF has one node for each point $\boldsymbol{v}_{p}(t)=\left(\boldsymbol{x}_{p}(t), t\right)$ along some path, for $t=1, \ldots, F$, and one binary random variable $\nu_{p}(t)$ per node. The neighborhood of $\boldsymbol{v}_{p}(t)$ is the set of points $\boldsymbol{v}_{q}(t)$ with $q \neq p$ and $\left\|\boldsymbol{v}_{p}(t)-\boldsymbol{v}_{q}(t)\right\| \leq \Delta$ for some small fixed $\Delta$ (spatial neighborhood), plus the two points $\boldsymbol{v}_{p}(t-1)$ and $\boldsymbol{v}_{p}(t+1)$ that are temporally adjacent to $\boldsymbol{v}_{p}(t)$ along path $p$ (temporal neighborhood).

Each node in the MRF is associated with a binary $o b$ served visibility flag $\hat{\nu}_{p}(t)$ computed from the data as follows. Path points in each frame are scored by their consistency, which measures how little a patch around $\boldsymbol{v}_{p}(t)$ changes as it is transported by the current estimates of paths near $\boldsymbol{v}_{p}(t)$ to (i) a few frames before and after time $t$, and (ii) the anchor frame $\tau_{p}$ for path $p$, similar to LME. The controlling path at $\boldsymbol{v}_{p}(t)$ is the most consistent path through the spatial neighborhood of $\boldsymbol{v}_{p}(t)$. Let now

$$
\bar{d}_{p q}=\frac{1}{F} \sum_{t=1}^{F}\left\|\boldsymbol{x}_{p}(t)-\boldsymbol{x}_{q}(t)\right\|
$$

be the average distance between two paths, and let $p^{*}$ be the controlling path at $\boldsymbol{v}_{p}(t)$. Then, the observed visibility 
$\hat{\nu}_{p}(t)$ is defined as follows (see also Figure 2):

$$
\hat{\nu}_{p}(t)=\left\{\begin{array}{ll}
1 & \text { if } \bar{d}_{p p^{*}} \leq 4 \text { pixels } \\
0 & \text { otherwise }
\end{array} .\right.
$$

In words, a path $p$ is observed to be visible at $\boldsymbol{v}_{p}(t)$ when it either coincides with ( $p=p^{*}$ so that $\bar{d}_{p p^{*}}=0$ ) or is nearly parallel $\left(\bar{d}_{p p^{*}} \leq 4\right)$ to the controlling path $p^{*}$ at $\boldsymbol{v}_{p}(t)$.

The observed visibility flags $\hat{\nu}_{p}(t)$ influence the (hidden) visibility flags $\nu_{p}(t)$ through a data term in the MRF. Let

$$
\Delta I_{p}(t)=\Psi\left(I\left(\boldsymbol{c}_{p}, t\right)-I\left(\boldsymbol{c}_{p}, \tau_{p}\right)\right)
$$

be the same per-path, per-frame measure of intensity consistency used in (3). We define the following average measure of intensity change along the visible portion of path $p$ :

$$
\Delta_{p}=\frac{\sum_{t=1}^{T} \hat{\nu}_{p}(t) \Delta I_{p}(t)}{\sum_{t=1}^{T} \hat{\nu}_{p}(t)} .
$$

For correctly estimated paths, this measure reflects variations of intensity caused by unmodeled effects such as image noise or global illumination changes, rather than by occlusions. Given these definitions, the data term of the MRF is defined as follows:

$$
\begin{aligned}
& D\left(\nu_{p}(t)=1\right)=\Delta I_{p}(t)+\lambda_{L}\left(1-\hat{\nu}_{p}(t)\right) \\
& D\left(\nu_{p}(t)=0\right)=\Delta_{p}+\lambda_{L} \hat{\nu}_{p}(t) .
\end{aligned}
$$

The terms with multiplier $\lambda_{L}$ bias estimated visibility values $\nu_{p}(t)$ toward observed values $\hat{\nu}_{p}(t)$. Setting a point to be visible incurs the additional charge $\Delta I_{p}(t)$, equal to the change in intensity between anchor and current point. Setting a point to be invisible incurs the additional charge $\Delta_{p}$ that accounts for the fact that intensity variations may be caused by other than occlusions.

The weights on edges between the random variables of the MRF encourage both temporal and spatial consistency among visibility values. Specifically, a penalty

$$
V\left(\nu_{p}(t), \nu_{p}(t+1)\right)=\lambda_{T}\left|\nu_{p}(t)-\nu_{p}(t+1)\right|
$$

is added between temporally adjacent neighbors to discourage changes of visibility along a path. The weight on an edge between spatial neighbors is

$$
V\left(\nu_{p}(t), \nu_{q}(t)\right)=\lambda_{S} w_{p q}(t)\left|\nu_{p}(t)-\nu_{q}(t)\right|
$$

with

$$
w_{p q}(t)=\frac{e^{-\left(\frac{\Delta I_{p q}(t)+\Delta I_{p q}}{\sigma^{2}}\right)}}{\bar{d}_{p q}+\epsilon}
$$

where $\epsilon>0$ prevents division by zero. In this expression,

$$
\begin{aligned}
& \Delta I_{p q}(t)=\left(I\left(\boldsymbol{c}_{p}, t\right)-I\left(\boldsymbol{c}_{q}, t\right)\right)^{2} \\
& \Delta I_{p q}=\left(I\left(\boldsymbol{c}_{p}, \tau_{p}\right)-I\left(\boldsymbol{c}_{q}, \tau_{q}\right)\right)^{2} .
\end{aligned}
$$

In words, $\Delta I_{p q}(t)$ measures difference in appearance between paths in a single frame, and $\Delta I_{p q}$ measures a similar difference between anchor points. The combined effect of these two terms is to push discontinuities in visibility closer to intensity boundaries, and the division by $\bar{d}_{p q}$ reduces the spatial discontinuity penalty between unrelated paths.

Finally, we clamp enough visibility values to 1 to ensure that every pixel in the sequence has a visible path through it. Specifically, we make all anchor points visible, $\nu_{p}\left(\tau_{p}\right)=1$, and we also force $\nu_{p}(t)=1$ if $\bar{d}_{p p^{*}}<\sqrt{2}$. This assignment guarantees that at least one visible path goes through every pixel because $\bar{d}_{p^{*} p^{*}}=0$. We roll the pairwise cost for each edge incident to a clamped node into the unary cost for the other node of that edge.

\section{Preliminaries}

Before we solve for motion and visibility, we select basis paths and an initial set of anchors, paths, and visibility flags.

\subsection{Finding the basis paths}

Basis paths are obtained by first tracking a sparse set of feature points with a frame-to-frame tracker [13]. This yields several tracks, that is, paths that do not necessarily extend through the entire sequence. These tracks are supplemented with those formed by concatenating optical flow vectors between consecutive frames [18], as described in more detail in Section 4.2, where we do the same to initialize a dense set of paths.

For some sequences, several tracks may extend from first to last frame. PCA can then yield a basis whose size $K$ is determined by adding principal components until the reconstruction residual for the input tracks is below, e.g., 2 pixels.

In general, however, occlusions and tracking failures make tracks start late and end early, leading to a matrix of track coordinates with missing entries. We iterate between matrix factorization with missing data [6] and a compaction step that associates tracks corresponding to the same world point [16]. If needed, a user can be asked to correct mistakes in data association. We scale path coordinates so that the mean per-path motion between frames is one pixel.

\subsection{Initialization}

To cover every pixel in a video sequence with paths, we need to create a number of paths of the same order of the number of visible points in the sequence. To this end, we form an initial set of paths by placing anchor points at every pixel in the first and last frames in the sequence, and supplement these with additional anchors in regions that are not yet covered by some path. More specifically, we start by defining path fragments we call temporal superpixels with the procedure described by Sundaram et al. [18]. We first concatenate optical vectors into tracks, which we 


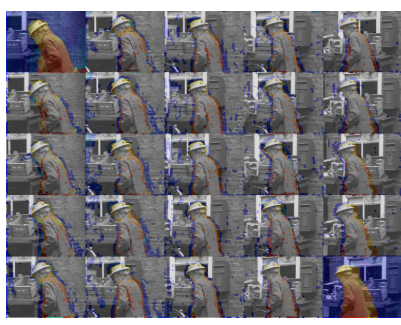

(a)

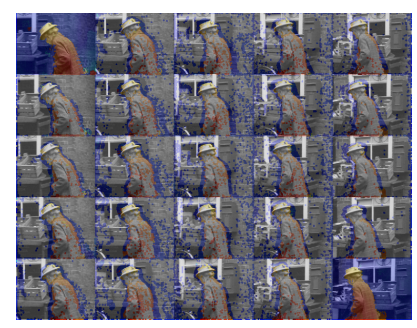

(b)
Figure 3: Anchor point selected during initialization (a) and at convergence (b). Colors other than gray denote anchor points, and similar colors denote similar sets of path coefficients. Note the improved segmentation of Miss Marple after convergence.

break when the optical flow field fails a forward-backward consistency check or when the point is too close to a motion boundary (equations (5) and (6) from [18], respectively). To prevent merging foreground and background tracks, we create a thin empty buffer around the regions where tracks terminate. If a superpixel thus created extends over several frames, we replace it by an entire path whose coefficients are computed by projecting the superpixel onto the path basis. If the superpixel is too short, we copy the coefficients of the path with the greatest intensity consistency over a few frames among existing, nearby, parallel paths. The temporal extent of superpixels provides an initial estimate for the visibility flags.

Every temporal superpixel that extends to the first or the last frame yields an initial path anchored in that frame. The remaining superpixels are said to be covered if their paths differ by an average of less than two pixels per frame from an existing path. New paths are formed only for superpixels that are not yet covered, and their anchor points are placed in the last frame of the superpixel. Figure 3 a shows the anchor points selected in this way for the marple 7 sequence. Colors other than gray are anchors, and similar colors correspond to similar sets of path coefficients.

After this initialization stage, the energy functions defined in Section 3 are minimized by the algorithms described in Section 5. This can result in the insertion of additional anchor points. Figure $3 \mathrm{~b}$ shows the color-coded anchor points after convergence.

\section{Optimization}

Starting with the paths and visibility flags constructed as described in Section 4.2, we interleave two steps during optimization: a combinatorial optimization step finds visibility flags $\nu_{p}(t)$ for the current path estimates, and a continuous optimization step updates path coefficients $\boldsymbol{c}_{p}$ given the current visibility estimates. In the process, we add an-

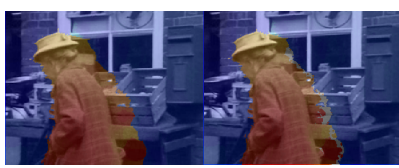

(a) Without regrouping.

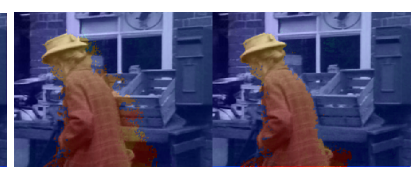

(b) With regrouping.
Figure 4: Effect of path regrouping. Motion estimates are shown using the same color scheme as in Figure 3. The first image in each pair shows the solution after the first round of optimization; the second shows results at convergence. Regrouping (b) recovers from a poor local optimum with incorrect estimates for the motion of the occluded background.

chor points until every pixel in the sequence has at least one path through it, and remove anchors of invisible paths. We stop when the maximum change in every path falls below one pixel in every frame.

The initial path estimates are often poor along occlusion boundaries, because visibility is not yet accounted for. Because of this, we heuristically regroup paths between each combinatorial and continuous step to let foreground and background vie for paths between them.

We now describe the continuous step, path regrouping, combinatorial step, anchor management, and termination.

Continuous step. We update path coefficients by minimizing the energy function (2) via trust-region Newton Conjugate Gradients optimization [14]. This method only requires computing vectors of the form $H \boldsymbol{v}$ where $H$ is the Hessian, rather than the very large but sparse $H$ itself. The sparsity pattern of $H$ changes over time because the coupling coefficients $\alpha_{p q}$ in equation (5) depend in turn on the path coefficients. When computing successive conjugate gradients, we treat the terms $\alpha_{p q}$ as constants-a good approximation for small path perturbations-and recompute them between full descent steps.

Path regrouping. After 40 descent steps, we allow paths to copy their coefficients and visibility flags from one of their neighbors if doing so improves the path's fit to data. Specifically, path $p$ copies from path $q$ if $\nu_{q}\left(\tau_{p}\right)=1, \tau_{p} \neq \tau_{q}$, $d_{p q}\left(\tau_{p}\right)<\Delta$, path $q$ is visible for at least half the frames, and the copy improves the data fit for $p$ the most. Figure 4 illustrates the benefits of this step on the marple 7 sequence. Combinatorial step. Visibility flags are updated after path regrouping by using graph cuts $[2,12]$ to compute the MAP estimate for the MRF in Section 3.2. The energy function is amenable to this method as the edge costs (12) satisfy

$$
\begin{aligned}
V(0,0)+V(1,1) & \leq V(0,1)+V(1,0) \\
0 & \leq V(0,1)+V(1,0) .
\end{aligned}
$$

Anchor management. When the maximum change in any path in any frame is less than one pixel, we check that every 
pixel in the video has a visible path through it. If not, we add new anchor points to fill voids and resume optimization. Newly inserted paths copy their initial parameters from the closest visible path.

Anchors on paths that are invisible everywhere except at the anchor itself (which is always visible) are deleted. These one-point paths occur when visibility estimation correctly identifies an outlier with an incorrect path estimate.

Termination. Optimization terminates when all path estimates change by less than a pixel in every frame and all pixels in the video have a path through them.

\section{Results}

We evaluate the performance of our technique on five real sequences of increasing complexity, all with large motions and significant occlusions. The popular flowerbed (29 frames) and a new sequence with a truck driving behind a road sign (33 frames) contain only rigid motion. The three with non-rigid motion are from the Berkeley motion segmentation dataset [5]: 60 frames from marple1, 72 frames from marple8, and 25 frames from marple7. The marple 7 and flowerbed sequences are the same as those evaluated in LME. Figure 5 shows sample frames; the full sequences are included in the supplemental material.

\subsection{Qualitative evaluation}

For a qualitative evaluation, we use our motion results to warp all frames to a selected frame. This creates a motioncompensated video that should appear static except for regions that are occluded in a particular frame, and that we paint white. Figure 5 shows the last frame aligned to the first frame, and viceversa, for all sequences.

We compare our results to LME paths and LDOF trajectories using implementations provided by their authors. We also ran two-frame optical flow between all pairs of frames, but even methods specifically aimed at large displacements [4] failed for distant frames. Figure 6 shows examples of mistakes made by competing methods.

\subsection{Quantitative evaluation}

It is difficult to get reliable ground truth paths for realistic sequences. Synthetic datasets [7] do not preserve associations across occlusions. Manual labeling for real sequences is painstaking and unreliable, particularly for complex motions or low-texture regions.

Instead, we measure the degree to which intensities remains constant along computed paths as a proxy for performance. We define the all-path interpolation error (APIE)

$$
\mathrm{APIE}=\sqrt{\frac{1}{N} \sum_{p} \frac{\sum_{t} \nu_{p}(t)\left(I\left(\boldsymbol{c}_{p}, t\right)-I\left(\boldsymbol{c}_{p}, \tau_{p}\right)\right)^{2}}{\sum_{t} \nu_{p}(t)}}
$$
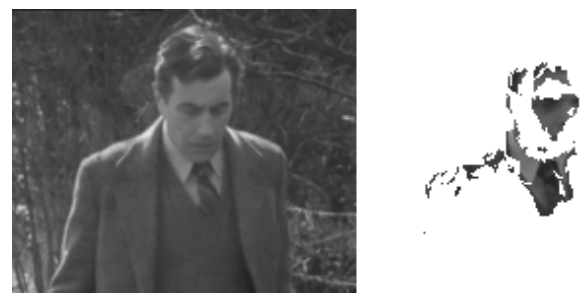

(a) LDOF warp of last frame to first frame of marple1.

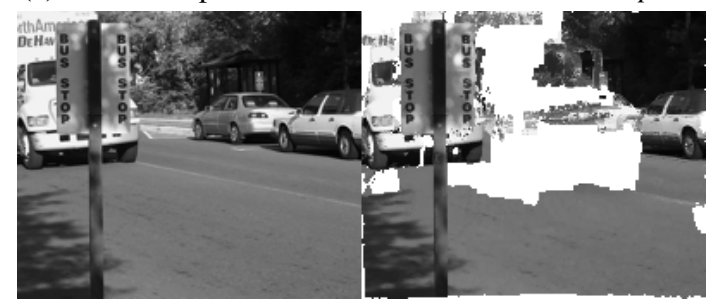

(b) LME warp of 20th frame to first frame of truck.

Figure 6: Examples of mistakes made by competing methods visible when warping to align with the first frame. White regions mark missing correspondences (left) or occluded pixels (right). LDOF trajectories do not bridge occlusions and suffer from drift. Few correspondences remain between the first and last frame of the marplel sequence, and the details of the motion of the man's face have been missed, leading to misalignment. (Misalignment is obvious in the accompanying video; see the supplemental material.) LME paths bridge occlusions, but they require that all occluders be visible in either the first or the last frame. The front of the truck is not visible in either the first or last frame, so it is not tracked as a potential occluder. As a result, portions of the background are incorrectly marked as visible and included in the warp back to the first frame with their appearance replaced by the missed occluding object.

where $N$ is the number of paths. Even on perfect paths, APIE would measure the correctness of the brightness constancy assumption, and would be nonzero in general.

Table 1 reports the APIE for different methods for each sequence, computed with intensity values in $[0,255]$. LDOF trajectories do not directly report visibility; the correspondences for trajectories after occlusions are simply missing. These entries are ignored as if they had $\nu_{p}(t)=0$. We use the location and frame of the first observation of each trajectory as its reference appearance. LME paths are anchored either in the first or last frame and do report visibility values.

We aim to recover paths that maintain correspondence across occlusions. We measure our success by analyzing the average length of a path, defined as $\frac{1}{N} \sum_{p} \sum_{t} \nu_{p}(t)$. As can be seen in Table 1, the two methods that estimate visibility (our method and LME) return significantly longer paths on average as the result of their ability to detect disocclusions. 


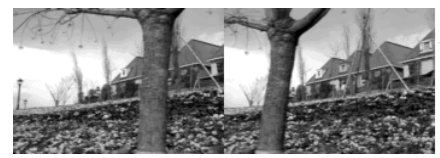

(a) Flowerbed. Two basis functions; 2.5 hours (29 frames).
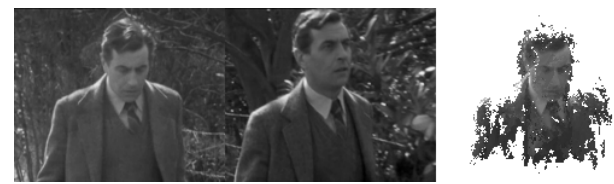

(c) Marple1. Eight basis functions; 65 hours (60 frames). Shadows cause problems for our occlusion detection step, so that not all of the man is recognized as being visible in both frames.

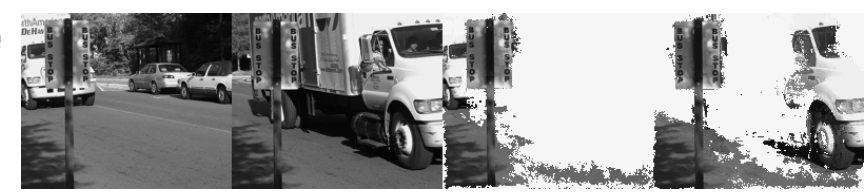

(b) Truck. Four basis functions; 20 hours (33 frames).

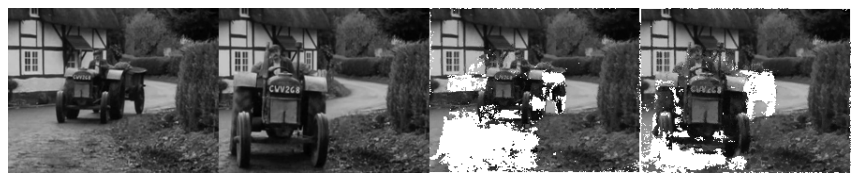

(e) Marple8. Eight basis functions; 68 hours (72 frames). Miss Marple (off-screen in both the first and last frames) walks past during the sequence, completely occluding the entire scene.

Figure 5: Results of our method. For each sequence, we show the first and last frames, followed by the last frame warped to align with the first frame, and vice versa. Regions detected as occluded in the source frame of the warp are marked in white. See supplemental material for the original videos and videos warped to the first, last, and middle frames. Solution times (rounded to nearest half-hour) exclude basis computation.

\begin{tabular}{|c|c|c|c|c|c|c|c|c|}
\hline \multirow{2}{*}{ Sequence } & \multirow{2}{*}{ Method } & \multirow{2}{*}{ APIE } & \multicolumn{2}{|c|}{ Path length (frames) } & \multicolumn{3}{|c|}{ Path density (pixels) } & \multirow{2}{*}{$\begin{array}{c}\% \text { pixels containing } \\
\text { visible paths }\end{array}$} \\
\hline & & & Mean & Std. dev. & 50th & 95 th & 99th & \\
\hline \multirow{3}{*}{ Flowerbed } & LDOF traj. & 13.97 & 11.2 & 10.5 & 0.47 & 8.5 & 15.2 & $66.6 \%$ \\
\hline & LME & 9.37 & 23.9 & 7.3 & 0.31 & 0.79 & 1.3 & $97.5 \%$ \\
\hline & Ours & 6.10 & 23.3 & 7.2 & 0.29 & 0.66 & 0.84 & $99.8 \%$ \\
\hline \multirow{3}{*}{ Truck } & LDOF traj. & 19.74 & 6.8 & 7.5 & 1.2 & 47.0 & 70.0 & $47.3 \%$ \\
\hline & LME & 17.82 & 23.4 & 7.4 & 0.39 & 1.9 & 4.4 & $88.6 \%$ \\
\hline & Ours & 9.80 & 22.0 & 9.0 & 0.27 & 0.65 & 0.86 & $99.8 \%$ \\
\hline \multirow{3}{*}{ Marple7 } & LDOF traj. & 7.84 & 6.7 & 6.4 & 0.47 & 6.9 & 13.3 & $69.0 \%$ \\
\hline & LME & 6.28 & 15.9 & 7.5 & 0.43 & 5.7 & 9.7 & $76.0 \%$ \\
\hline & Ours & 5.61 & 15.5 & 6.8 & 0.32 & 0.70 & 0.89 & $99.7 \%$ \\
\hline \multirow{2}{*}{ Marple1 } & LDOF traj. & 15.83 & 9.4 & 11.4 & 0.51 & 14.7 & 26.7 & $62.5 \%$ \\
\hline & Ours & 8.79 & 18.9 & 19.3 & 0.35 & 0.85 & 1.0 & $97.8 \%$ \\
\hline \multirow{2}{*}{ Marple8 } & LDOF traj. & 13.69 & 14.9 & 14.7 & 0.47 & 7.4 & 16.4 & $71.2 \%$ \\
\hline & Ours & 9.30 & 45.8 & 20.8 & 0.25 & 0.65 & 0.87 & $99.7 \%$ \\
\hline
\end{tabular}

Table 1: Solution quality metrics. APIE measures average intensity constancy along estimated paths (smaller is better, assuming the brightness constancy assumption holds). Path length is the number of frames in which a path is reported as visible (longer is typically better). Path density is computed by measuring the distance to the nearest visible path for each pixel. We report the 50th, 95th, and 99th percentiles (smaller is better), as well as the percentage of pixels with a visible path within a radius of 1 pixel (larger is better). The marple1 and marple 8 sequences were too large for LME to complete within a reasonable timeframe. We stopped computation after 72 hours when only a single iteration had completed.

Further, the average length of our paths tends to correspond to the length of the dominant occluder.

A key feature of our algorithm is the ability to compute the path for every visible point in a scene. We measure path density by computing the distance to the closest visible path for each pixel in the sequence. Table 1 reports the 
50th, 95th, and 99th percentile for each method, as well as the total percentage of pixels with a visible path within a distance of 1 pixel. LDOF trajectories leave many pixels unexplained because they are not initialized in low-texture areas. LME misses objects not visible in either the first or last frame of a sequence. In many sequences, these missed objects can account for a significant fraction of the scene. Our method explains over $97 \%$ of the pixels in every sequence.

\subsection{Parameter sensitivity}

Our technique uses a few parameters that could be tuned if desired. We selected settings for the parameters by hand considering the results on the flowerbed sequence only, and used the same values for all five sequences. We set $\lambda=1$, $\sigma=50$, and $\lambda_{L}=\lambda_{T}=\lambda_{S}=0.5$. We re-scale intensity values to $[0,1]$ for the combinatorial optimization step to match the range of the binary unknowns. In our experiments, we found that results were relatively insensitive to small changes in the values of $\lambda$ or $\sigma$, but were more sensitive to the values of the parameters for the occlusion detection step.

\section{Conclusion}

We introduced a method to compute extended video motion paths that explain every pixel of a video sequence. We regularize the solution by projection on a low-dimensional basis of motion paths, and can follow points through brief occlusions. In contrast with previous methods, we can handle occlusions wherever they occur. This is made possible by a new, non-variational formulation that allows for more realistic visibility and appearance constraints and is also more efficient than the variational approach. While we focused on grayscale video, the extension to color is straightforward.

Much work remains to be done. Video with many or highly deformable moving objects such as crowds or flags [9] may require nonparametric methods, and our solution may perhaps be used for initialization. Sparsityinducing priors on the path coefficients are an intriguing alternative, in which each path is allowed to use a small number of functions from a large basis. Processing even longer sequences than the one we can handle will require models of long-term, global changes in illumination, and separate motion bases for different video segments. How to splice together solutions from these segments is an open challenge, and so is greater computational efficiency.

\section{Acknowledgements.}

This work is supported by the Army Research Office under Grant No. W911NF-10-1-0387 and by the National Science Foundation under Grant IIS-10-17017.

\section{References}

[1] I. Akhter, Y. Sheikh, S. Khan, and T. Kanade. Trajectory Space: A Dual Representation for Nonrigid Structure from Motion. PAMI, 33(7):1442-1456, 2011. 2

[2] Y. Boykov and V. Kolmogorov. An experimental comparison of min-cut/max-flow algorithms for energy minimization in vision. PAMI, 26(9):1124-1137, 2004. 5

[3] C. Bregler, A. Hertzmann, and H. Biermann. Recovering non-rigid 3D shape from image streams. In CVPR, 2000. 2

[4] T. Brox and J. Malik. Large displacement optical flow. In CVPR, 2009. 2, 6

[5] T. Brox and J. Malik. Object Segmentation by Long Term Analysis of Point Trajectories. In ECCV, 2010. 6

[6] A. Buchanan and A. Fitzgibbon. Damped Newton algorithms for matrix factorization with missing data. In CVPR, 2005. 4

[7] D. J. Butler, J. Wulff, G. B. Stanley, and M. J. Black. A naturalistic open source movie for optical flow evaluation. In ECCV, 2012. 6

[8] J. Costeira and T. Kanade. A multibody factorization method for independently moving objects. IJCV, 29(3):159-179, 1998. 2

[9] R. Garg, L. Pizarro, D. Rueckert, and L. Agapito. Dense multi-frame optic flow for non-rigid objects using subspace constraints. In $A C C V, 2010.2,8$

[10] R. Garg, A. Roussos, and L. Agapito. Robust TrajectorySpace TV-L1 Optical Flow For Non-rigid Sequences. In EMMCVPR, 2011. 2

[11] M. Irani. Multi-frame correspondence estimation using subspace constraints. IJCV, 48(3):173-194, 2002. 2

[12] V. Kolmogorov and R. Zabin. What energy functions can be minimized via graph cuts? PAMI, 26(2):147-159, 2004. 5

[13] B. Lucas and T. Kanade. An iterative image registration technique with an application to stereo vision. In IJCAI, 1981. 2, 4

[14] J. Nocedal and S. J. Wright. Numerical Optimization. Springer Series in Operations Research. Springer Verlag, 2nd edition, 2006. 5

[15] S. Ricco and C. Tomasi. Dense Lagrangian Motion Estimation with Occlusions. In CVPR, 2012. 1, 2

[16] S. Ricco and C. Tomasi. Simultaneous Compaction and Factorization of Sparse Image Motion Matrices. In ECCV, 2012. 4

[17] P. Sand and S. Teller. Particle Video: Long-range motion estimation using point trajectories. IJCV, 80(1):72-91, 2008. 2

[18] N. Sundaram, T. Brox, and K. Keutzer. Dense point trajectories by GPU-accelerated large displacement optical flow. In ECCV, 2010. 2, 4, 5

[19] C. Tomasi and T. Kanade. Shape and motion from image streams under orthography: a factorization method. IJCV, 9(2):137-154, 1992. 2

[20] L. Torresani and C. Bregler. Space-time tracking. In ECCV, 2002. 2

[21] J. Weickert and C. Schnörr. Variational optic flow computation with a spatio-temporal smoothness constraint. Journal 
of Mathematical Imaging and Vision, 14(3):245-255, 2001.

2 